\title{
Synergistic Effect of Combining Titanosilicate and 1-Ethyl-3-Methylimidazolium Acetate in Mixed Matrix Membranes for Efficient $\mathrm{CO}_{2}$ Separation
}

\author{
By M.M. López Guerrero ${ }^{1}$, C. Casado-Coterillo², A. Irabien ${ }^{2}$
}

\begin{abstract}
The separation and capture of $\mathrm{CO}_{2}$ from these sources is becoming important for greenhouse emission. The membrane-based separation process use to remove $\mathrm{CO}_{2}$ takes advantages in energy efficient and environmentally friendly aspects and has been recognized as an important technology for $\mathrm{CO}_{2}$ capture and gas separation. The novel mixed matrix membranes (MMMs) were fabricated by incorporating microporous titanosilicate ETS-10 and a highly $\mathrm{CO}_{2}$ absorbent ionic liquid , [EMIM][Ac] into a Chitosan (CS) matrix to improve $\mathrm{CO}_{2}$ separation performance, and were prepared, characterized and tested for $\mathrm{CO}_{2}$ and $\mathrm{N}_{2}$. The solubility values show that the $\mathrm{CO}_{2}$ solubility increases upon addition of ETS-10 particles. The $\mathrm{N}_{2}$ solubility in the ETS10/[EMIM][Ac]/CS was reduced, while $\mathrm{CO}_{2}$ solubility remained constant. FT-IR spectra revealed a good interaction between the components in the MMMs. Subtle differences in the intensity and position of all individual bands, were observed in the region between 3600 and $2700 \mathrm{~cm}^{-1}$ and 1700 and $900 \mathrm{~cm}^{-1}$. These indicated bonding of the components in the film, confirming the good interaction existing among the components, and that may account for the higher flexibility of the hybrid membrane materials imparted to both CS and ETS-10/CS MMMs, , due to the singular interaction between CS and [EMIM][Ac].
\end{abstract}

Keywords: $\mathrm{CO}_{2}$ solubility, $\mathrm{CO}_{2}$ separation, microporous titanosilicate ETS-10, Chitosan, mixed matrix membranes (MMMs).

\section{Introduction}

Despite the significant progress in the development of new renewable energy in recent years, fossil fuels likely still occupy a dominant position in the worldwide energy source for the future (Conti 2013). Because of this situation, the separation and capture of $\mathrm{CO}_{2}$ is becoming important for greenhouse emission and global warming, with over 30 billion tonnes of global $\mathrm{CO}_{2}$ emissions per year in last years (McGee 2011). Economic and effective techniques for $\mathrm{CO}_{2}$ removal are desirable and have a great interest. Conventional methods usually involve cost and complicated equipment and high energy consumption. Compare with traditional methods, the membrane based separation process has advantages in energy efficient and environmentally friendly aspects and has been recognized as an important technology for $\mathrm{CO}_{2}$ capture and gas separation (D’Alessandro et al. 2010; Chung et al. 2007; Zhang et al. 2013; Huang et al. 2012).

Membrane-based gas separation has been postulated to compete with absorption in terms of energy requirement when $\mathrm{CO}_{2}$ content in the feed is larger than $20 \%$ due to its low energy consumption, easy operation and low maintenance (Jiang et al. 2006). Membranes are usually classified as polymeric, inorganic, and more recently, mixed matrix membranes (MMMs). Transport through a dense-polymeric membrane usually takes place through the solutiondiffusion mechanism in three steps: the selective component adsorbs in the membrane, where

${ }^{2}$ Department of Chemical and Biomolecular Engineering, Universidad de Cantabria, Santander, Spain. 
diffuses through and the component desorbs from the other side, due to the low pressure kept at the permeate side.

Polymer membranes are low cost, cheaper than inorganic membranes and have good mechanical stability at high pressure. Nevertheless, polymeric membranes suffer from either low selectivity or permeability, and usually show an inverse relationship between permeability and selectivity (Zhang et al. 2013). The selectivity is regulated by the polymer molecular structure that allows preferential passing of certain gas molecules based on their sizes, normally represented by their kinetic diameters. The permeability is largely controlled by the gas solubility. The main parameter that determines the solubility is the ability of the penetrant gases to condense (Zhang et al. 2013).

Mixed matrix membranes (MMMs) are heterogeneous materials formed by the combination of an organic polymer continuous matrix and inorganic material dispersed phase, with the aim of obtaining a well-dispersed heterogeneous mixture of synergistic properties and overcoming the accepted trade-off between permeability and selectivity for gas separation membranes (Zhang et al. 2013; Robeson, 2008). Due to the chemical potential difference across membrane takes place the permeation. The permeability is usually calculated as the product of solubility (S) and diffusivity (D).

The main challenge in MMMs is the adhesion between dispersed and continuous phases. To improve adhesion, there are several strategies which have been studied, such as functionalization of the inorganic particles prior to introduction into the polymer matrix, or the incorporation of an ionic liquid in the membrane matrix (Hudiono et al. 2010; Hao et al. 2013). In a previous work, we proposed a similar approach but using a non-toxic ionic liquid, a biopolymer and a microporous zeo-type material prepared without organic surfactant (Casado-Coterillo et al. 2014b).

The components used were chitosan, a microporous titanosilicate and an ionic liquid. Chitosan $(\mathrm{CS})$ is a continuous polymer, poly $[\beta(1,4) 2$-amino-2deoxy-D-glucopyranose]. CS is a linear polysaccharide obtained by the deacetylation of chitin, which is a natural polymer, cheap, biodegradable, biocompatible, non-toxic, unique structure, interesting properties and hydrophilic. Due to their good film-forming ability, they can be used to form edible films which may provide an alternative to synthetic materials (Tharanathan 2003). High hydrophilicity makes CS prone to effective barriers against $\mathrm{O}_{2}, \mathrm{CO}_{2}$ and aromas, but their barrier properties against water are poorer which makes CS membrane effective for $\mathrm{CO}_{2}$ separation due to the high $\mathrm{CO}_{2}$ solubility in water (Liu et al. 2008). Furthermore, CS membranes have been well studied for $\mathrm{CO}_{2}$ separation (Ito et al. 1997; El-Azzami and Grulke 2008; El-Azzami and Grulke 2009). Its mechanical stability has nevertheless been tried to improve by coating on a porous polysulfone support (Kai et al. 2008), organic chemical crosslinking (Xiao, Feng and Huang 2007), and physical mixing with zeolite particles (Casado-Coterillo et al. 2014).

The mechanical stability of CS has been tried to improve by coating on a porous polysulfone support (Kai et al. 2008), organic chemical crosslinking (Xiao et al. 2007), and physical mixing with zeolite particles (Casado-Coterillo et al. 2014). Facilitated transport in the solid matrix is expected to increase the stability as well, and CS, because of the weak acid-base interactions between $\mathrm{CO}_{2}$ and water molecules and the amino groups in the chains, has potential to enhance the electrostatic interactions among permeating molecules and the functional groups in the polymer by introducing appropriate materials. Mixed matrix membranes (MMMs) have been prepared by filling the CS matrix with 5 wt. \% of ETS-10 titanosilicate with synergistic transport properties in pervaporation and gas separation (Casado-Coterillo et al. 2014a, b).

The structure of the microporous titanosilicate ETS-10 is made of orthogonal $\mathrm{TiO}_{6}$ octahedra and $\mathrm{SiO}_{2}$ tetrahedra linked by oxygen atoms shared in the corners. Ti atoms in a six-coordinated state have two negative charges balanced by $\mathrm{Na}^{+}$and $\mathrm{K}^{+}$(Casado et al. 2009). The high cation exchange capacity is what makes ETS-10 very interesting in adsorption (Tiscornia et al. 2010), 
catalysis, and membrane separation processes (Tiscornia et al. 2010). ETS-10 can be synthesised in different sizes including nano-scale (Casado et al. 2009), which may be homogeneously dispersed in a polymer providing this with its intrinsic characteristics. Ionic liquids (ILs) combining good and tuneable solubility properties with negligible vapour pressure and good thermal stability have recently received much attention as green solvents and $\mathrm{CO}_{2}$ absorbents in supported liquid membrane contactors (Santos et al. 2014). The $\mathrm{CO}_{2}$ solubility is higher when acetate is the anion and the shorter length of the cation, and 1-ethyl-3-methylimidazolium acetate, [EMIM][Ac], the room temperature ionic liquid (IL) with the highest reported $\mathrm{CO}_{2}$ solubility (Blath et al. 2012), as well as non-reported toxicity (Alvarez-Guerra and Irabien 2011), was chosen for the proof-of-concept of this work. A good interaction with CS is expected since it has been reported as a good solvent for polysaccharides (Ding et al. 2012), because of the strong H-bonds forming with the $\mathrm{OH}$ groups in the polymer chain. $\mathrm{CS}$ and chitin have been reported to enhance the $\mathrm{CO}_{2}$ solubility of low absorbing [bmim] [Cl] because the ionic liquid is able to alter the $\mathrm{H}$ bonds in the polymer chains, thus freeing amino groups that become available for $\mathrm{CO}_{2}$ absorbing sites (Xie, Zhang and Li 2006). Novel MMMs composed of CS, [EMIM][Ac]/CS, ETS-10/CS and ETS-10/[EMIM][Ac]/CS, with the small loading of dispersed filler of $5 \mathrm{wt}$. \% with respect to the continuous polymer matrix, were prepared and tested for $\mathrm{CO}_{2}$ and $\mathrm{N}_{2}$ permeation (CasadoCoterillo et al. 2014b). In this work, the factors affecting membrane morphology such as adhesion, interaction among components, and thermal, chemical, and mechanical resistance have been extensively explored by further FT-IR experiments and discussion complemented those of the previous work.

\section{Experimental}

Chitosan (coarse ground flakes and powder, Sigma-Aldrich, Madrid, Spain) with a deacetylation degree higher than $75 \mathrm{wt} \%$ and high viscosity in $1 \mathrm{wt} \%$ acetic acid/water was used. $\mathrm{TiO}_{2}$-anatase (powder, $99.8 \mathrm{wt} \%$, Aldrich, Madrid, Spain) and sodium silicate solution (27 wt \% $\mathrm{SiO}_{2}, 8 \mathrm{wt} \% \mathrm{Na}_{2} \mathrm{O}$, Merck, Barcelona, Spain) as Ti and Si source, respectively, for the ETS-10 crystal synthesis, and 1-ethyl-3-methylimidazolium acetate, [EMIM][Ac], (99\%, Sigma Aldrich) were used as purchased.

\subsection{Preparation of ETS-10}

In a typical synthesis, $35.06 \mathrm{~g}$ of parent gel with molar composition 5.6 $\mathrm{SiO}_{2}: 1 \mathrm{TiO}_{2}: 4.6$ $\mathrm{Na}_{2} \mathrm{O}: 1.9 \quad \mathrm{~K}_{2} \mathrm{O}: 137 \quad \mathrm{H}_{2} \mathrm{O}$ were poured into a Teflon-lined autoclave and submitted to hydrothermal synthesis at $230{ }^{\circ} \mathrm{C}$ for $24 \mathrm{~h}$. The autoclave was then removed from the oven and quenched under cold tap water to room temperature. The solid was washed and centrifuged at least 3 times, and dried at $100{ }^{\circ} \mathrm{C}$ overnight to recover about $2.8 \mathrm{~g}$ of final product. This product has a particle size of $\mathrm{a}=\mathrm{b}=0.32 \pm 0.06 \mu \mathrm{m}$ and $\mathrm{c}=0.41 \pm 0.22 \mu \mathrm{m}$ and a BET surface area of $253 \pm 7 \mathrm{~m}^{2} / \mathrm{g}$.

\subsection{Preparation of membranes.}

The preparation of the pure CS and CS-based MMMs has been reported elsewhere (Casado-Coterillo et al. 2014). In a typical synthesis, first, CS 2 wt. \% solutions were first dissolved in 2 wt. \% acetic acid (glacial, Panreac) aqueous solutions under stirring at $80{ }^{\circ} \mathrm{C}$ for 24 $\mathrm{h}$ at reflux conditions. The CS solution obtained was filtered to remove insoluble impurities and degassed in an ultrasonic bath before $10 \mathrm{~mL}$ on a polystyrene Petri dish and evaporating at room temperature for 2-3 days. CS membranes were then removed from the Petri dish. A $15.55 \mathrm{~cm}^{2}$ membrane was cut from the film for gas permeation and neutralized in $1 \mathrm{M} \mathrm{NaOH}$ and rinsed with abundant distilled water and dried at $4{ }^{\circ} \mathrm{C}$ before $\mathrm{CO}_{2}$ and $\mathrm{N}_{2}$ permeation experiments in 
order to ion-exchange the $\mathrm{NH}_{3}{ }^{+}$functional groups of the polymer matrix. ETS-10 particles were first dispersed in distilled water (proportion 1:100 wt/wt) in an ultrasound bath for $10 \mathrm{~min}$ at room temperature. Then, CS solution $(10 \mathrm{~mL})$ was added and treated in ultrasound bath for 15 min until a homogenous white dispersion was obtained and cast as described above. IL/CS membranes were prepared with a nominal 5 wt. \% IL loading with respect to CS. In a typical synthesis: $0.042 \mathrm{~g}$ of IL (97 wt. \%, Sigma-Aldrich, Madrid, Spain) were added to the $10 \mathrm{~mL}$ CS solution and stirred overnight before casting in a similar manner as the pure CS membranes. For the three-component ETS-10/[EMIM][Ac]/CS MMMs, the preparation method was similar to that employed for ETS-10/CS MMMs, using the [EMIM][Ac]/CS mixture as continuous phase.

\subsection{Equipment}

IR Spectra were recorder on a Perkin Elmer Spectrum 100 FTIR spectrometer (Perkin Elmer, Concord, Canada) with a resolution of $4 \mathrm{~cm}^{-1}$ and 32 accumulations. Solid samples were measured by using potassium bromide pellets. The solid samples were dried at $100{ }^{\circ} \mathrm{C}$ for at least $2 \mathrm{~h}$ and grinded for $5 \mathrm{~min}$, the concentrations for the samples were $10 \mathrm{wt}$. \% approximately. Per sample sample/KBr mixture (approx. 10/100 wt/wt and $100 \mathrm{mg}$ in total) were made. Also one $\mathrm{KBr}$ was made as a reference.

The liquid sample was measured using optical transmission cell, having windows compatible with infrared wavelengths. All cells used had planar windows and the liquid samples were measured dripping several drops of the sample between two thin pieces of glass and sandwiching it. In both cases, the absorbance values were determined between 4000 and $450 \mathrm{~cm}^{-1}$.

The mechanical resistance of the membranes was measured by the tensile strength and the elongation at break of 5-10 of $5 \mathrm{~mm}$ wide samples of the membrane materials in a Universal Testing Machine (Zwick/Roell, Ulm, Germany) with a head load up to $2.5 \mathrm{kN}$ and $5 \mathrm{~mm} / \mathrm{min}$.

\section{Results and Discussion}

\subsection{FT-IR spectra}

In order to characterize the initial materials, a spectrum of pure chitosan was recorded (Fig.1a). The main bands appearing in the spectrum of chitosan (CS) powder is due to stretching vibrations of $\mathrm{OH}$ groups in the range from $3750 \mathrm{~cm}^{-1}$ to $3000 \mathrm{~cm}^{-1}$ and $\mathrm{C}-\mathrm{H}$ bond in $-\mathrm{CH}_{2}(2930$ $\left.\mathrm{cm}^{-1}\right)$ and $-\mathrm{CH}_{3}\left(2875 \mathrm{~cm}^{-1}\right)$ groups, respectively. Bending vibrations of methylene and methyl groups were also visible at $1380 \mathrm{~cm}^{-1}$ and $1460 \mathrm{~cm}^{-1}$, respectively (Mano et al., 2003). The range of $1680-1480 \mathrm{~cm}^{-1}$ was related to the vibrations of carbonyl bonds $(\mathrm{C}=\mathrm{O})$ of amide group CONHR (secondary amide, $1660 \mathrm{~cm}^{-1}$ ) and to the vibrations of amine group $\mathrm{NH}_{2}, 1580 \mathrm{~cm}^{-1}$ (Ruel-Gariépy and Leroux. 2006). The spectra in the range from $1160 \mathrm{~cm}^{-1}$ to $1000 \mathrm{~cm}^{-1}$ have been analysed by many authors who attribute vibrations appearing in this range to CO group (Duarte et al. 2002; $\mathrm{Xu}$ et al. 2005). The band located near $1150 \mathrm{~cm}^{-1}$ is related to asymmetric vibrations of $\mathrm{CO}$ in oxygen bridge. Resulting from deacetylation of chitosan. The bands near $1080-1025 \mathrm{~cm}^{-1}$ are attributed to $\mathrm{CO}$ of the ring $\mathrm{COH}, \mathrm{COC}$ and $\mathrm{CH}_{2} \mathrm{OH}$ (Duarte et al. 2002; Shigemasa et al. 1996; Nunthanid et al. 2001). 


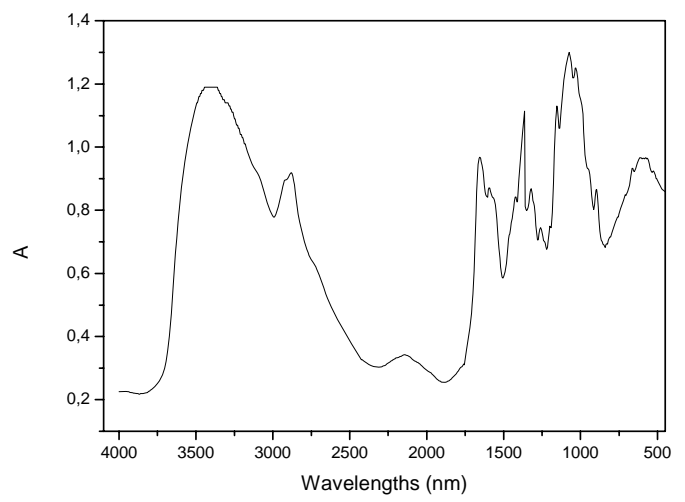

Figure 1a. Chitosan FT-IR spectrum.

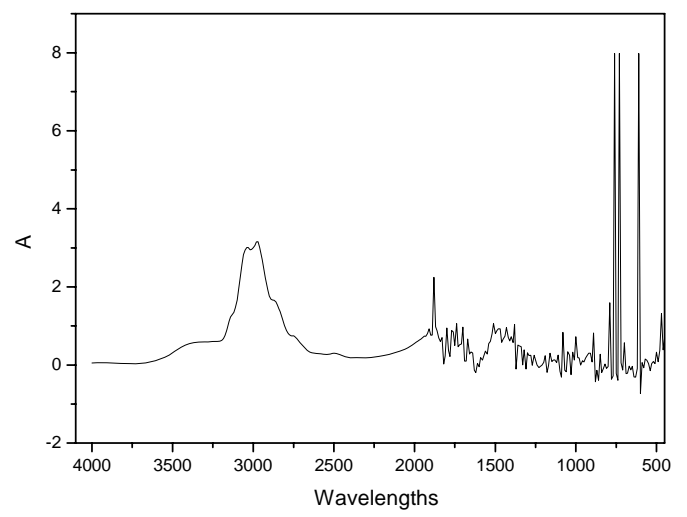

Figure 1b. EMIM Ac FT-IR spectrum

On the other hand, the [EMIM]-Ac ionic liquid shows a characteristic absorption pattern between 1700 and $600 \mathrm{~cm}^{-1}$, Fig. 1b. In the fingerprint region, that is, in the spectral range below $1500 \mathrm{~cm}^{-1}$, numerous peaks overlapping with each other are observed. Nevertheless, several characteristic features can be assigned to their vibrational modes. The main contributions found in the spectral range between 500 and $1000 \mathrm{~cm}^{-1}$ can be attributed to the [EMIM] cation. An in-plane symmetric bending mode of the ring, with contribution from a $\mathrm{CH}_{3}(\mathrm{~N}) \mathrm{CN}$ stretching mode, is found at 600 $\mathrm{cm}^{-1}$. Asymmetric bending of the ring $\mathrm{HCCH}$ group shows up at $756 \mathrm{~cm}^{-1}$, and a ring bending vibration from the $\mathrm{NC}(\mathrm{H}) \mathrm{N}$ group, with contribution from the $\mathrm{CCH}$ bending, is found at $845 \mathrm{~cm}^{-1}$. CC stretching is observed at $958 \mathrm{~cm}^{-1}$. Substantial contributions from the acetate anion are found in the spectral range between 1000 and $2000 \mathrm{~cm}^{-1}$ (along with further lines from the [EMIM] cation). A ring in-plane asymmetric stretching, with contributions from the $(\mathrm{N}) \mathrm{CH}_{2}, \mathrm{CH}_{3}(\mathrm{~N}) \mathrm{CN}$, and CC stretching, results in a strong peak at $1169 \mathrm{~cm}^{-1}$. The $\mathrm{C}-\mathrm{CH}_{3}$ stretching vibration from acetate is found at $1254 \mathrm{~cm}^{-1}$, whereas the bending mode of $\mathrm{CH}_{3}$ is present at $1425 \mathrm{~cm}^{-1}$. In between (at 1389 $\mathrm{cm}^{-1}$ ), another strong line attributed to [EMIM] is observed, where a ring in-plane asymmetric stretching, a $\mathrm{CH}_{2}(\mathrm{~N})$ bending, a CC stretching, a $\mathrm{CH}_{2}(\mathrm{~N})$ stretching, and a $\mathrm{CH}_{3}(\mathrm{~N}) \mathrm{CN}$ stretching overlap. The $\mathrm{C}=\mathrm{O}$ stretching band is found at $1566 \mathrm{~cm}^{-1}$ (this signal is normally located at around 
$1700 \mathrm{~cm}^{-1}$ in non-ionic carbonyl compounds). This frequency shift can be explained by both a delocalization of the electrons in the ionic acetate compound and substantial intermolecular interactions with the [EMIM] cation, which influence the vibrational structure.

A number of comparatively weak overlapping $\mathrm{CH}$ stretching modes is observed in the range 2900-3200 $\mathrm{cm}^{-1}$, whereas a broad band corresponding to the intermolecular O-H vibrations caused by hydrogen bonds is present between 3200 and $3700 \mathrm{~cm}^{-1}$ (Kiefer et al 2008).

It is well-known that in the region of $3800-3000 \mathrm{~cm}^{-1}$ the water vibration is present. It is important to note that dry [EMIM][Ac] does not have absorptions in this region. This is very interesting, since we have observed that the introduction of the ionic liquid in the chitosan matrix greatly reduces the water swelling of the chitosan matrix, thus improving the flexibility of the films. These might help correlating FTIR to the data on Table 1.

The molecules produce a broad band in the region around $3450 \mathrm{~cm}^{-1}$. The strengthening of $\mathrm{O}-\mathrm{H}$ stretching vibration band is due to the interactions between [EMIM] [Ac] and water. The water would easily be absorbed due to forming strong H-bonds with the anion of [EMIM] [Ac].

In this spectrum the vibrational frequencies of 1-ethyl-3-methylimidazolium cation, acetate anion, and C1 and C2 conformers of their ion pair are shown. The IR bands at 1005, 1120, 1170 and $1647 \mathrm{~cm}^{-1}$ agree with the vibrational structure of the $\mathrm{C} 1$ conformer. This suggests that the $\mathrm{C} 1$ conformer is the major contributor to the vibrational structures observed in the experimental spectra. C2-H13 stretching frequency by $1197 \mathrm{~cm}^{-1}$ in the $\mathrm{C} 1$ conformer with respect to the free cation value $3203 \mathrm{~cm}^{-1}$. This dramatic shift is attributed to the strong $\mathrm{C}-\mathrm{H} \cdots \mathrm{O}$ interaction between the cation and anion considered (Nilesh et al. 2009).

The $1647 \mathrm{~cm}^{-1}$ band observed in the IR spectrum is assigned to the O9-C10 local stretching mode combined with N3-C4-H14 rocking vibrations (Nilesh et al. 2009).

Turning to ethyl and methyl groups of the cation, bending vibrations are generally in good agreement with measurements, for instance, the bands appearing at frequencies 1449 and 1454 $\mathrm{cm}^{-1}$ are assigned to H19-C7-H2O twisting vibrations. Likewise, bands at 1388 and $1393 \mathrm{~cm}^{-1}$ are attributed to the intense mode arising from $\mathrm{H} 21-\mathrm{C} 8-\mathrm{H} 22$ rocking vibrations. While the assignment is very reasonable, we nevertheless point out that the IR peak at $1388 \mathrm{~cm}^{-1}$ could be assigned instead to the $\mathrm{C} 10-\mathrm{C} 12$ stretching vibrations of the acetate anion in the ion pair (Dumal et al. 2009).

When two or more substances are mixed, physical blends versus chemical interactions are reflected by changes in characteristic spectra peaks. Furthermore, the increased flexibility imparted to the MMMs by the introduction of the IL was attributed to the singular interaction between CS and IL, and due to this fact, the FT-IR spectra of the membranes were measured and studied (Figure 2).

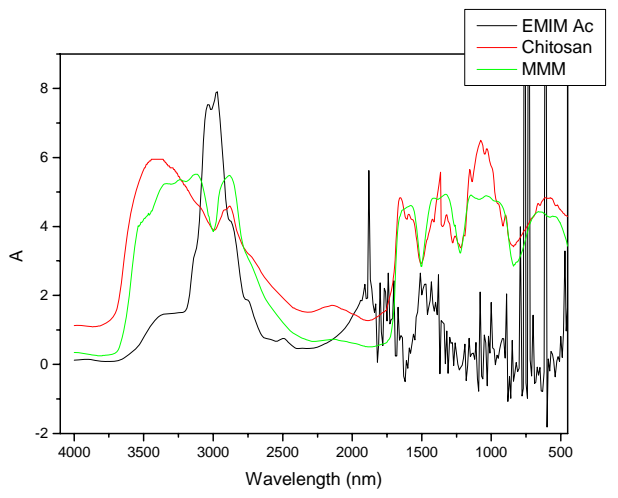

Figure 2. FT-IR of MMMs, CS and EMIM Ac. 
Subtle differences in the intensity and position of all individual bands, observed both in the region between 3600 and $2700 \mathrm{~cm}^{-1}$ and between 1700 and $900 \mathrm{~cm}^{-1}$, clearly indicated bonding of the components of the film. In the first region, between 3600 and $2700 \mathrm{~cm}^{-1}$, as a result of the formation of the three-component film, the absorption bands at 3280 and $2940 \mathrm{~cm}^{-1}$ of MMM decreased in intensity and moved by 5 and $10 \mathrm{~cm}^{-1}$, respectively, to lower wave numbers. The CS and 3-component ETS-10/IL/CS membranes demonstrated a broad band in the range 3600$2700 \mathrm{~cm}^{-1}$, attributed to $\mathrm{NH}$ and $\mathrm{OH}$ vibrations, whereas the broad band at around $2940 \mathrm{~cm}^{-1}$ in CS is shifted to lower wave numbers due to film formation.

The modified band around $2940 \mathrm{~cm}-1$ might reflect these conformational changes of the polymer chains.

In the second region, between 1700 and $900 \mathrm{~cm}^{-1}$, the amide I, amide II and amide III bands decreased in intensity due to the formation of the three-component film. That trend followed former findings revealed by Taravel and Domard (1995). Moreover, a band at $1377 \mathrm{~cm}^{-1}$ of chitosan, in the spectrum of the MMM could be recognized as a shoulder of the peak at $1410 \mathrm{~cm}^{-}$ 1 . The shift of the latter by $10 \mathrm{~cm}^{-1}$ towards higher wave numbers compared with its location in the spectrum of the film pointed at participation of the -COO. This confirms the good interaction existing among the components involved on MMM preparation, and that may account for the higher flexibility of the hybrid membrane materials imparted to both CS and ETS-10/CS MMMs, as explained above regarding mechanical properties of the membranes in Table 1.

\subsection{Other membrane properties.}

Other properties of the MMMs were evaluated in a previous work and correlated the observations of FTIR experiments discussed above, regarding the synergic effect of the use of ETS-10 particles and [EMIM][Ac] ionic liquid as fillers to the CS matrix. The crystallinity, $\chi$, of the CS-based membrane samples was also calculated from the FTIR spectra, using the ratio of the absorbance at 1423 and $890 \mathrm{~cm}^{-1}$, respectively. The crystallinity increased monotonously upon addition of [EMIM][Ac], ETS-10 and both to the CS polymer matrix, as reported for other MMMs prepared from semi-crystalline polymers (Shen and Luca, 2012), where this phenomenon was attributed to the role of inorganic fillers as nucleating agents. The crystallinity is important in membrane analysis since only the amorphous part of the polymer is contributing to the gas separation.

The tensile strength diminishes upon addition of [EMIM][Ac]and ETS-10 particles. This was attributed to plasticization of the polymer matrix reflected by the large increase on the value of the elongation at break, for the [EMIM][Ac]/CS with respect to CS membranes. The introduction of [bmim] $\left[\mathrm{CF}_{3} \mathrm{SO}_{3}\right]$ IL in semi crystalline Pebax polymers (Bernardo et al. 2012) caused a decrease in the elongation at break that has not been observed here. On the other hand, the elongation at break of the CS-based membranes decreases upon addition of ETS-10 particles. This is due to the rigidification of the organic polymer (Xu et al. 2011) by the addition of inorganic fillers.

Table 1. MMMs properties (Casado-Coterillo et al. 2014)

\begin{tabular}{|c|c|c|c|c|c|c|}
\hline $\begin{array}{l}\text { Membrane material } \\
\text { composition }\end{array}$ & $\begin{array}{c}\text { Thickness } \\
(\mu \mathrm{m})\end{array}$ & $\begin{array}{l}\text { Tensile } \\
\text { strength } \\
\text { (MPa) }\end{array}$ & $\begin{array}{c}\text { Elongation } \\
\text { at break } \\
(\%)\end{array}$ & $\chi(-)$ & $\begin{array}{c}S\left(\mathrm{CO}_{2}\right) \\
\mathrm{cm}^{3}(\mathrm{STP}) / \mathrm{cm}^{3} \\
\mathrm{Hg}\end{array}$ & $\begin{array}{c}S\left(\mathrm{~N}_{2}\right) \\
\mathrm{cm}^{3}(\mathrm{STP}) / \mathrm{cm}^{3} \\
\mathrm{Hg}\end{array}$ \\
\hline $\mathrm{CS}$ & $121.9 \pm 3.9$ & $31.6 \pm 7.4$ & $18.5 \pm 7.4$ & 0.14 & 0.08 & 0.005 \\
\hline [EMIM & $128.0 \pm 3.6$ & $16.1 \pm 11.0$ & $40.4 \pm 11.0$ & 0.18 & 0.51 & 0.026 \\
\hline ETS-1 & $130.0 \pm 4.5$ & $24.3 \pm 4.9$ & $14.4 \pm 9.4$ & 0.28 & 0.08 & 0.003 \\
\hline ETS-10/[EMIM][Ac]/CS & $168.0 \pm 5.0$ & $19.9 \pm 5.0$ & $36.2 \pm 3.0$ & 0.34 & 0.07 & 0.002 \\
\hline
\end{tabular}

The solubility values were calculated from the thermogravimetrical sorption experiments. As it was said previously, CS shows a high hydrophilicity that makes CS prone to enhanced $\mathrm{CO}_{2}: \mathrm{N}_{2}$ 
perm-selectivity, ETS-10 shows a high exchange capacity, which is very interesting in adsorption, and [EMIM][Ac] shows good solubility properties. The combination of these three components increases the solubility of $\mathrm{CO}_{2}$ due to the $\mathrm{H}$-bonds in the polymer chains, thus freeing amino groups that become available for $\mathrm{CO}_{2}$ absorbing sites (Xie et al. 2006). The solubility increases upon addition of ETS-10, due to its adsorption affinity toward $\mathrm{CO}_{2}$. On the other hand, the $\mathrm{N}_{2}$ solubility decreases in ETS-10/CS MMMs, due to the molecular sieve effect of thenanoporous titanosilicate. The $\mathrm{N}_{2}$ solubility in MMMs was decreased, because of the incorporation of $\mathrm{CO}_{2}-$ soluble IL, while $\mathrm{CO}_{2}$ solubility remained constant, compared with the two-component ETS10/CS MMM (Casado-Coterillo et al. 2014).

\section{Conclusion}

FT-IR spectra revealed a good interaction between the components in the MMMs. The FT-IR are proven to be the efficient methods for the characterization of the interaction between [EMIM] [Ac], ETS-10 and CS. The shift of the vibrational frequency has been used to correlate the relative strength of $\mathrm{H}$-bond. The $\mathrm{CO}_{2}$ solubility increased upon addition of both the [EMIM][Ac] and ETS-10 adsorbent, the mechanical strength was enhanced and the selectivity was six times higher than pure CS membrane, thus leading to a synergistic effect on the membrane properties as a result of the good interaction between the components.

\section{Acknowledgements}

Financial support from the Spanish Ministry of Economy and Competitiveness (MINECO) under project CTQ2012-31229 is gratefully acknowledged.

\section{References}

Alvarez-Guerra, M.; Irabien, A. (2011) Design of ionic liquids: An ecotoxicity (Vibrio fischeri) discrimination approach. Green Chem., 13: 1507-1516.

Bernardo, P.; Jansen, J.C.; Bazzarelli, F.; Tasselli, F.; Fuoco, 2012, A. Gas transport properties of Pebax (R) /room temperature ionic liquid gel membranes. Sep. Purif. Technol. 97: 73-82.

Blath, J.; Deubler, N.; Hirth, T.; Schiestel, T. (2012) Chemisorption of carbon dioxide in imidazolium based ionic liquids with carboxylic anions. Chem. Eng. J., 181-182, 152-158.

Casado, C.; Amghouz, Z.; García, J.R.; Boulahya, K.; González-Calbet, J.M.; Téllez, C.; Coronas, J. (2009) Synthesis and characterization of microporous titanosilicate ETS-10 obtained with different Ti sources. Mater. Res. Bull. 44: 1225-1231.

Casado-Coterillo, C.; Andrés, F.; Téllez, C.; Coronas, J.; Irabien, A. (2014) Synthesis and characterization of ETS-10/chitosan nanocomposite materials for pervaporation. Sep. Sci. Technol. 49: 1903-1909.

Casado-Coterillo, C.; López-Guerrero, M. d. M. and Irabien A. (2014) Synthesis and characterisation of ETS10/Acetate-based ionic liquid/chitosan mixed matrix membranes for $\mathrm{CO}_{2} / \mathrm{N}_{2}$ separation. Membranes, 2014, 4 (2): 287-301.

Chung, T.S., Jiang, L.Y., Li, Y., Kulprathipanja, S., (2007) Mixed matrix membranes (MMMs) comprising organic polymers with dispersed inorganic fillers for gas separation, Prog. Polym. Sci. 32: 483-507.

D’Alessandro, D.M., Smit, B., Long, J.R., (2010) Carbon dioxide capture: prospects for new materials, Angew. Chem. Int. Ed. 49: 6058-6082.

Ding, Z.-D.; Chi, Z.; Gu, W.-X.; Gu, S.-M.; Liu, J.-H.; Wang, H.J. (2012) Theoretical and experimental investigation on dissolution and regeneration of cellulose in ionic liquid. Carbohydr. Polym. 89: 716.

Duarte, M.L., Ferreire, M.C., Marvao, M.R., Roh, J. (2002) An optimised method to determine the degree of acetylation of chitin and chitosan by FTIR spectroscopy. Int. J. Biol. Macromol. 31: 1-8.

Dumal, N. R., Kim, H. J, Kiefer, J. 2009 Molecular interactions in 1 ethyl 3 methylimidazolium acetate ion pair: a density functional study. J. Phys Chem. A. 113: 10397-10404.

El-Azzami, L.A.; Grulke, E.A. (2008) Carbon dioxide separation from hydrogen and nitrogen by fixed facilitated transport in swollen chitosan membranes. J. Membr. Sci. 323: 225-234. 
El-Azzami, L.A.; Grulke, E.A. (2009) Parametric study of CO fixed carrier facilitated transport through swollen chitosan membranes. Ind. Eng. Chem. Res. 48, 894-902.

Freeman, B.D. (1999) Basis of permeability/selectivity trade-off relations in polymeric gas separation membranes. Macromol32, 375-380.

Hao, L.; Li, P.; Yang, T.; Chung, T.S. (2013) Room temperature ionic liquid/ZIF-8 mixed-matrix membranes for natural gas sweetening and post-combustion $\mathrm{CO}_{2}$ capture. J. Membr. Sci. 436: 221-231.

Huang, A., Wang, N., Kong, C., Caro, J., (2012) Organosilica-functionalized zeolitic imidazolate framework ZIF-90 membrane with high gas-separation performance, Angew. Chem. Int. Ed. 51: 1055110555.

Hudiono, Y.C.; Carlisle, T.K.; Bara, J.E.; Zhang, Y.; Gin, D.L.; Noble, R.D (2010). A three-component mixed-matrix membrane with enhanced $\mathrm{CO}_{2}$ separation properties based on zeolites and ionic liquid materials. J. Membr. Sci. 350: 117-123.

Conti J., International energy Outlook, DOE/EIA-0484, U.S. Independent Statistics and Analysis, U.S. Energy Information Adminsitration, Washintong DC, 2013. On the web; www.eia.gov//ieo/, last consulted on 30/03/2015.

Ito, A.; Sato, M.; Anma, T. (1997) Permeability of $\mathrm{CO}_{2}$ through chitosan membrane swollen by water vapor in feed gas. Angew. Makromol. Chem. 248: 85-94. 14.

Jiang, L.Y., Chung, T.S., Kulprathipanja, S. (2006) Fabrication of mixed matrix hollow fibers with intimate polymer-zeolite interface for gas separation. AIChE J. 52: 2898-2908.

Kai, T.; Kouketsu, T.; Duan, S.; Kazama, S.; Yamada, K. (2008) Development of commercial-sized dendrimer composite membrane modules for $\mathrm{CO}_{2}$ removal from flue gas. Sep. Purif. Technol. 63: 524-530. 17.

Kiefer, J., Obert, K., Bösmann, A., Seeger, T., Wasserscheid, P., Leiertz. A. (2008) Quantitative analysis of alpha-D-glucose in an ionic liquid by using infrared spectroscopy. Chem Phys Chem. 9: 13171322.

Liu, L.; Chakma, A.; Feng, X. (2008) Gas permeation through water-swollen hydrogel membranes. J. Membr. Sci., 310: 66-75.

Mano, J.F., Koniarova, D., Reis, R.L., (2003) Thermal properties of thermoplastic starch/synthetic polymer blends with potential biomedical applicability. medicine. Mater. Sci. Mater. Med. 14: 127-136.

Ruel-Gariépy, E. and Leroux J.C. Chitosan: a natural polycation with multiple applications. In: Marcessault, R.H., Ravenelle, F., Zhu, XX. Polyssacharides for drug delivery and pharmaceutical applications, American Chemical Society, Washington 2006: 243-259.

McGee, M. $\mathrm{CO}_{2}$ now, http://CO2now.org/Current-CO2/CO2-now/global-carbon-emissions.html, 2011, last consulted on 29/03/2015.

Nilesh, R. D., Hyung, J.K., Johannes, K. (2009), Molecular Interactions in 1-Ethyl-3-methylimidazolium Acetate Ion Pairs: A density Functional Study, J Phys. Chem. 113, 10397-10404.

Nunthanid, J., Puttipipatkahachorn, S, Yamamoto, K., Peck, G.E. (2001), Physical properties and molecular behavior of chitosan films, Drug Dev. Ind. Pharm. 27: 143-157.

Robeson, L.M. (2008) The upper bound revisited. J. Membr. Sci. 320, 390-400.

Santos, E.; Albo, J.; Irabien, A. (2014) Acetate based supported ionic liquid membranes (SILMs) for $\mathrm{CO}_{2}$ separation: Influence of the temperature. J. Membr. Sci., 452: 277-283.

Shen, Y., Luca. A.c., (2012) Preparation and characterization of mixed matrix membranes based on PVDF and three inorganic fillers (fumed nonporous silica, zeolite 4A and mesoporous MCM-41) for gas separation. Chem. Eneg. J.,122: 427-435.

Shigemasa, Y., Matsuura, H., Sasmoto, H., Sashiwa, H., (1996) Evaluation of Different Absorbance Ratios from Infrared Spectroscopy for Analyzing the Degree of Deacetylation in Chitin. Int. J. Biol. Macromol. 18: 237-242.

Taravel M.N., Domard, A. (1995) Collagen and its interaction with chitosan. Biomaterials 18: 865-871.

Tharanathan., R. N. (2003) Biodegradable films and composite coatings: past, present and future. Trend. Food Sci. Technol. 14: 71-78.

Tiscornia, I.; Irusta, S.; Prádanos, P.; Téllez, C.; Coronas, J.; Santamaría, J. (2007) Preparation and characterization of titanosilicate Ag-ETS-10 for propylene and propane adsorption. J. Phys. Chem. C. 111: 4702-4709.

Tiscornia, I.; Kumakiri, I.; Bredesen, R.; Téllez, C.; Coronas, J. (2010) Microporous titanosilicate ETS-10 membrane for high pressure $\mathrm{CO}_{2}$ separation. Sep. Purif. Technol. 73: 8-12. 
Xiao, S.; Feng, X.; Huang, R.Y.M. (2007) Trimesoyl chloride crosslinked chitosan membranes for $\mathrm{CO}_{2} / \mathrm{N}_{2}$ separation and pervaporation dehydration of isopropanol. J. Membr. Sci. 306: 36-46.

Xie, H.; Zhang, S.; Li, S. (2006) Chitin and chitosan dissolved in ionic liquids as reversible sorbents of $\mathrm{CO}_{2}$. Green Chem., 8: 630-633.

Xu, D.; Loo, L.S.; Wang, K. Characterization and diffusion behaviour of chitosan-POSS composite membranes. J. Appl. Polym. Sci. 2011, 122, 427-435.

Xu, Y., Kim, K., Hanna, M., Nag, D., (2005) Chitosan-starch composite films preparation and characterization. Ind. Crop. Prod. 21: 185-192

Zhang, C., Xiao, Y., Liu, D., Yang, Q., Zhong, C., (2013) A hybrid zeolitic imidazolate framework membrane by mixed-linker synthesis for efficient $\mathrm{CO}_{2}$ capture, Chem. Commun. 49: 600-602.

Zhang, Y., Sunarso, J., Liu, S., Wang, R. (2013) Current status and development of membranes for $\mathrm{CO}_{2} / \mathrm{CH}_{4}$ separation: a review, Int. J. Greenh. Gas Cont. 12: 84-107. 